1 Title:

\title{
2 Why SARS-CoV-2 Omicron variant is milder? A single high-frequency \\ 3 mutation of structural envelope protein matters.
}

\section{Authors:}

5 Bingqing Xia ${ }^{1,2,}$ *, Yi wang ${ }^{1,3}$, Xiaoyan $\operatorname{pan}^{4}$, Xi Cheng ${ }^{1,2}$, Hongying $\mathrm{Ji}^{1}$, Xiaoli Zuo ${ }^{1}$,

$6 \quad$ Jia $\mathrm{Li}^{1,2}$, Zhaobing Gao ${ }^{1,2, *}$

$7 \quad{ }^{1}$ CAS Key Laboratory of Receptor Research, Stake Key Laboratory of Drug Research,

8 Shanghai Institute of Materia Medica, Chinese Academy of Sciences, 555 Zuchongzhi

9 Road, Shanghai, 201203, China.

$10{ }^{2}$ University of Chinese Academy of Sciences, 19A Yuquan Road, Beijing, 100049,

11 China.

${ }^{3}$ State Key Laboratory of Medical Neurobiology, Institutes of Brain Science, Fudan

13 University, Shanghai 200032, China.

$14{ }^{4}$ State Key Laboratory of Virology, Wuhan Institute of Virology, Center for Biosafety

15 Mega-Science, Chinese Academy of Sciences, Wuhan, 430071, China.

16 These authors contributed equally: Bingqing Xia, Yi wang

$17 *$ Corresponding author. Email: zbgao@simm.ac.cn (Z.G.), xiabingqing@simm.ac.cn 18 (B.X.). 


\section{Abstract:}

SARS-CoV-2 Omicron variant is highly transmissible and extensive morbidity, which

21 has raised concerns for antiviral therapy. In addition, the molecular basis for the attenuated pathogenicity and replication capacity of Omicron remains elusive. Here, we report for the first time that a high-frequency mutation T9I on 2-E of SARS-CoV-2 variant Omicron forms a non-selective ion channel with abolished calcium permeability and reduced acid sensitivity compared to the WT channel. In addition, T9I caused less cell death and a weaker cytokine production. The channel property changes might be responsible for the Omicron variant releases less efficiently and induces a comparatively lower level of cell damage in the infected cells. Our study gives valuable insights into key features of the Omicron variant, further supporting 2-E is a promising drug target against SARS-CoV-2 and providing critical information for the COVID-19 treatment. 


\section{Mian Text:}

Since November 2021, a new SARS-CoV-2 variant (B.1.1.529) emerged in South Africa and was designated as the fifth variant of concern (VOC), named as Omicron ${ }^{1,}$ 2. Omicron variant is highly transmissible and extensive morbidity, which has raised concerns for antiviral therapy. Omicron has largest number $(>30)$ of substitutions, deletions, or insertions with mutations frequency higher than $1 \%$ in the spike protein (S). Mutation studies in the spike RBD domain have provided a plausible explanation for altered transmissibility and antibody resistance ${ }^{3-5}$. Recent reports revealed Omicron variant induces a longer cycle for virus shed, attenuates replication capacity, and produces substantially attenuated lung pathology, indicating that the pathogenic ability of Omicron variant is indeed milder ${ }^{6-8}$. However, the molecular basis for the attenuated pathogenicity and replication capacity remains elusive. The envelope protein of SARSCoV-2 (2-E) forms a homo-pentameric cation channel that is important for virus pathogenicity $^{9,10}$. It has been demonstrated that 2-E channel alone is sufficient to induce cell death, provoke cytokine storm and even cause acute respiratory distress syndrome (ARDS)-like damages in vivo. Its inhibitor exhibits excellent antiviral activity in vivo ${ }^{9}$. Therefore, we focus on the 2-E mutations of Omicron variant.

Three high quality E protein sequences of Omicron variants (B.1.1.529, BA1 and BA2) in the CNCB database as of January 18th, 2022 (https://ngdc.cncb.ac.cn/ncov/) were comprehensively analyzed and a mutation 2-E $\mathrm{E}^{\mathrm{T} 9 \mathrm{I}}$ (T9I) caught our attention. Compared with the original Wuhan reference strain, among more than one hundred identified 2-E mutations in Omicron variant, T9I shows a much higher frequency than the rest 
mutations. In addition, the mutation probability of T9I in all statistical samples is higher than $99.5 \%$. For the most common Omicron strain B.1.1.529, the mutation probability is $100 \%$ (Fig.1a, b). According to the solved NMR structure, T9 locates at the top of the transmembrane domain (TMD) of 2-E proteins ${ }^{10}$. The influence of T9I has not been studied yet completely.

First, we asked whether T9I still retains channel activity. We purified T9I protein and reconstituted the protein on Planar Lipid Bilayer (BLM) as previously described ${ }^{9}$ (Supplementary Fig.S1). The observed T9I-induced typical single-channel currents supported that T9I was able to form ion channels also as wild-type (WT) 2-E proteins ${ }^{9}$. However, the reversal potential of T9I channels was shifted to left, from $57 \mathrm{mV}$ to 3 $\mathrm{mV}$, under asymmetric $\mathrm{KCl}$ solutions. Similar reversal potential shifting was also detected in asymmetric $\mathrm{NaCl}$ solutions (Fig.1c.d, Supplementary Fig.S2). A reversal potential close to $0 \mathrm{mV}$ suggests that T9I may have lost its selectivity to cations. To determine whether T9I can permeate chloride, the channel activity of T9I was further examined in asymmetric 50:500 $\mathrm{mM}$ choline chloride solutions. The quaternary ammonium choline is not permeable to most cation channels and thus is always used to detect anion permeability. As expected, outward currents were indeed observed when the holding voltage potentials were higher than the theoretical reversal potential for chloride $\left(E_{\text {rev }}>70 \mathrm{mV}\right)$. Besides, WT channels are permeable to $\mathrm{Ca}^{2+}$. As shown in Figure S2c, for WT channels, the frequent and continuous inward potassium currents in the asymmetric 50:500 $\mathrm{mM}$ (trans: cis) $\mathrm{K}^{+}$solutions indicated that the 2-E channels were incorporated into the membranes. The membrane potential was then changed to 
$+65 \mathrm{mV}\left(\mathrm{K}^{+}\right.$reverse potential) to eliminate the $\mathrm{K}^{+}$currents. Intriguingly, outward steplike signals appeared when $20 \mathrm{mM}$ (final concentration) $\mathrm{Ca}^{2+}$ was added to the trans side. When the $\mathrm{Ca}^{2+}$ concentration increased to $20 \mathrm{mM}$, the outward currents increased. In contrast, under the identical conditions, T9I channels did not induce detectable calcium currents (Supplementary Fig.S2c). These results revealed that T9I owns a different ion selectivity and permeability from those of WT.

WT channels are $\mathrm{pH}$ sensitive ${ }^{9}$. The $\mathrm{pH}$ influences on T9I channels were evaluated on a same channel using titration strategy. After the channels incorporated into the membranes, $\mathrm{HCl}$ was titrated into either cis or trans side to alter the $\mathrm{pH}$ to 4 from 6 . Consistent to our previous results, reduction of $\mathrm{pH}$, either in cis or trans side, induced significant increase of amplitude and open probability of WT channels. In contrast, $\mathrm{pH}$ reduction in cis side failed to increase the channel activity of T9I channels. Although we didn't have direct evidences to explain how the threonine (T9) site is sensitive to $\mathrm{pH}$ changes, the structure of 2-E channels supported the residues (Glu7 and Glu8) around $\mathrm{T} 9$ were sensitive to $\mathrm{pH}$ change and their side chain carboxyl could be deprotonated at neutral $\mathrm{pH}$ and protonated at acidic $\mathrm{pH}^{10}$. It is reported that SARSCoV-2 traffics to the lysosomes for egress by lysosome deacidification, instead of using the conventional biosynthetic secretory pathway ${ }^{11}$. The E channels of coronavirus are localized to intracellular organelles, including lysosome and Golgi apparatus ${ }^{11,12}$. The cellular localization and expression of WT and T9I were assessed using immunocytochemistry and confocal microscopy. We found that both WT and T9I are co-localized with the lysosomal marker LAMP (Fig.1g, supplementary Fig.S3). Given 
the localization on lysosome, the influences of WT and T9I on the luminal pH of

101

102 lysosome were compared. Flow cytometric was used to analyze $\mathrm{pH}$ probe (pHluorin) ratio. This $\mathrm{pH}$ probe consists of green fluorescent protein (GFP) pHluorin molecule fused with lysosome-resident protein CD63. To generate a $\mathrm{pH}$ calibration curve, untransfected cells were subjected to treatment with buffers ranging from $\mathrm{pH} 5.5$ to 7.5 in the presence of the ionophores monensin and nigericin prior to flow analysis (Fig. $1 \mathrm{~h}$, i). Expression of WT channels robustly neutralized the lysosome $\mathrm{pH}$, whereas T9I channels exhibited less influences in luminal $\mathrm{pH}$, which was consistent with our electrophysiological results (Fig.1j). Therefore, the capability of deacidification of WT channels in lysosomes was significantly impeded by the mutation T9I. Based on above, whether the weakened capability of deacidification hampers virus releasing was examined. We transfected the Vero E6 cells with WT or T9I plasmids at day 1, then the cells were infected with SARS-CoV-2 virus at day 2. As determined by qRT-PCR, preexpression of mutant T9I significantly attenuated viral loadings compared with WT (Fig.1k). It was logic to deduce that some exogenous T9I subunits would co-assemble with the WT subunits originated from the virus and form heteromultimers with impaired $\mathrm{pH}$ sensitivity, resulting in less deacidification of lysosomes and less SARS-CoV-2 virus releasing.

Finally, the channel activity of the 2-E protein is a determinant of virulence. We used Annexin V/PI staining and CCK8 assays to evaluate the cell lethality of T9I in Vero E6 cells. We found that although the expression level of T9I was far exceeding the expression level of WT (Fig. 1 1), it caused similar cell death as WT did (Fig. $1 \mathrm{~m}$, 
supplementary Fig.S4a). Similarly, in the mouse macrophage cell line (RAW264.7), a very highegreater expression of T9I induced a comparable increase of inflammatory cytokines as WT did (Fig. 1n, Supplementary Fig.S4b). The correlation between the cell lethality and the expression level was further compared. As shown in Figure 1o, in comparison with WT, the killing cells capability of T9I was dropped around 150 times as well as the cytokines production. Notably, according to another latest study, the 2-E expression in Omicron variant was decreased approximately 3 -fold, which may further suppress the virulence of Omicron variants ${ }^{6}$. How much the weakened capabilities of killing cells and inducing cytokine production confer the milder clinical symptoms of Omicron warrant further investigation.

Overall, as SARS-CoV-2 infections are progressing, there are chances that virus accumulates new mutations. Among existed antiviral targets, different from spike protein, RNA-dependent RNA polymerase (Rdrp) and 3CL pro, which mediate virus entry, protein cleavage and viral replication, respectively, the envelope protein (2-E) majorly participating in the virus releasing ${ }^{13-15}$. Here we report for the first time that a high-frequency 2-E mutation T9I of SARS-CoV-2 variant Omicron forms a nonselective ion channel with abolished calcium permeability and reduced acid sensitivity compared to WT channels. In addition, T9I caused less cell death and a weaker cytokine production (Fig.1p). The channel property changes might be responsible for the Omicron variant releases less efficiently and induces a comparatively lower level of cell damage in the infected cells. According to 2-E NMR structures, although T9I is not located in the pore domain of the channel, it may alter the channel configuration via 
144 interacting with the amino acids at positions L12 and T11 (Supplementary Fig.S5). Our

145 study provides valuable insights into key features of the Omicron variant and critical

146 information for the COVID-19 treatment.

\section{Acknowledgement:}

148 We are grateful to the National Science Fund of Distinguished Young Scholars

149 (81825021), Fund of Youth Innovation Promotion Association (2019285), the National

150 Natural Science Foundation of China (81773707, 92169202), the National Key

151 Research and Development Program of China (2020YFC0842000), the National Key

152 Laboratory Program of China (LG202101-01-04), Fund of National Science and

153 Technology Major Project (2018ZX09711002-002-006) and the Hubei Science and

154 Technology Project (2020FCA003) for financial support.

155 Author contributions:

Z. G., and B. X. conceived designed the project. Z. G., J. L., and B. X designed the

157 experiments; Y. W. and B. X. performed the electrophysiological recordings; B. X.

158 carried out the cell-based assays; P. X. carried out the virus assays in vitro; Y. W purified

159 the proteins; H.Y. analyzed the sequence; all authors analyzed and discussed the data.

Z. G., B. X. wrote the manuscript. All authors read and approved the manuscript. 


\section{Reference:}

1 Karim SSA, Karim QA. Omicron SARS-CoV-2 variant: a new chapter in the COVID-

19 pandemic. Lancet (London, England) 2021; 398:2126-2128.

2 Organization WH. Classification of Omicron (B.1.1.529): SARS-CoV-2 Variant of C oncern. $<$ https://wwwwhoint/news/item/26-11-2021-classification-of-omicron (b11529) -sars-cov-2-variant-of-concern>.

3 Cao Y, Wang J, Jian F et al. Omicron escapes the majority of existing SARS-CoV-2 neutralizing antibodies. Nature 2021.

4 Cele S, Jackson L, Khoury DS et al. Omicron extensively but incompletely escapes Pfizer BNT162b2 neutralization. Nature 2021.

173 interferon antagonism but similar drug sensitivity in Omicron variant compared to Delta variant of SARS-CoV-2 isolates. Cell research 2022:1-3.

1777 Halfmann PJ, Iida S, Iwatsuki-Horimoto K et al. SARS-CoV-2 Omicron virus causes

178 attenuated disease in mice and hamsters. Nature 2022.

1798 Torjesen I. Covid-19: Peak of viral shedding is later with omicron variant, Japanese data suggest. BMJ (Clinical research ed) 2022; 376:089.

9 Xia B, Shen X, He Y et al. SARS-CoV-2 envelope protein causes acute respiratory distress syndrome (ARDS)-like pathological damages and constitutes an antiviral target. 
Structure and drug binding of the SARS-CoV-2 envelope protein transmembrane domain in lipid bilayers. Nature structural \& molecular biology 2020; 27:1202-1208. 1535.e1514.

12 Westerbeck JW, Machamer CE. The Infectious Bronchitis Coronavirus Envelope

Protein Alters Golgi pH To Protect the Spike Protein and Promote the Release of Infectious Virus. Journal of virology 2019; 93.

13 Yin W, Mao C, Luan X et al. Structural basis for inhibition of the RNA-dependent RNA polymerase from SARS-CoV-2 by remdesivir. Science (New York, NY) 2020; 368:1499-1504. its inhibitors. Nature 2020; 582:289-293. epitopes on SARS-CoV-2 spike. Nature 2020; 584:450-456. 

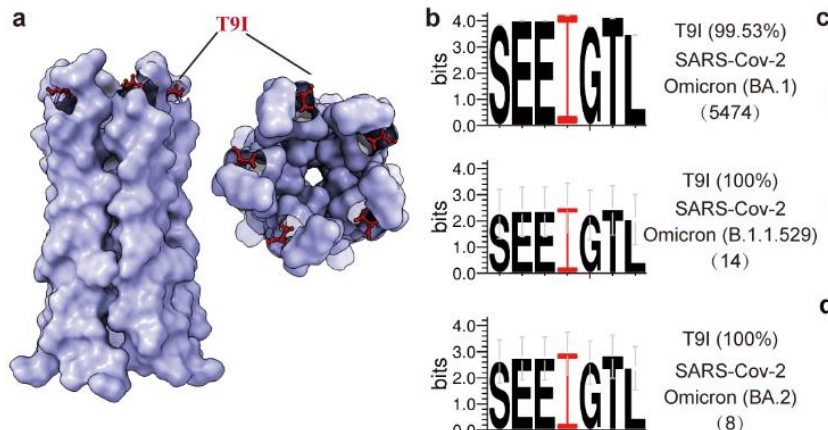

Transmembrane domain

WT ETGTLIVNSVLLFLAFVVFLLVTLAILTALR (8-38)

T9I E IGTLIVNSVLLFLAFVVFLLVTLAILTALR (8-38)
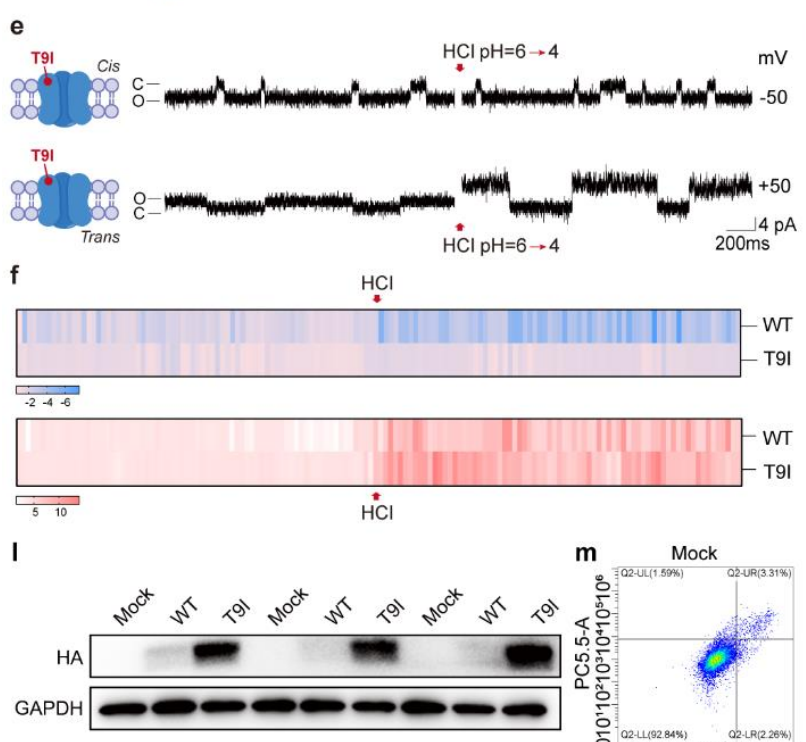

n

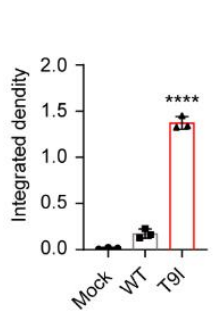

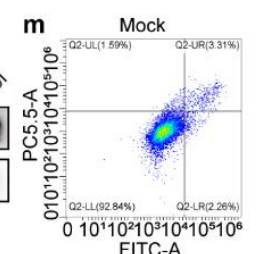

FITC
0

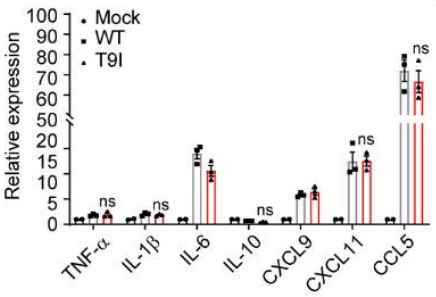

d

d
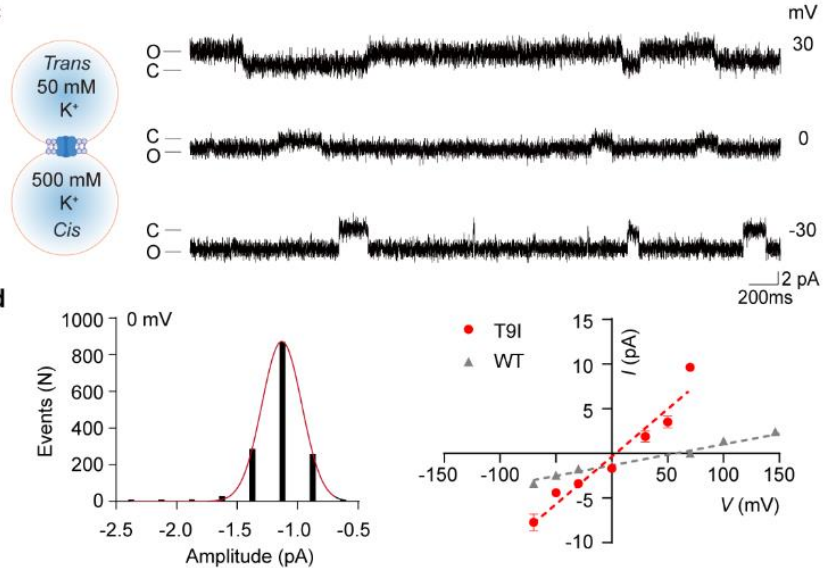

T9I-HA
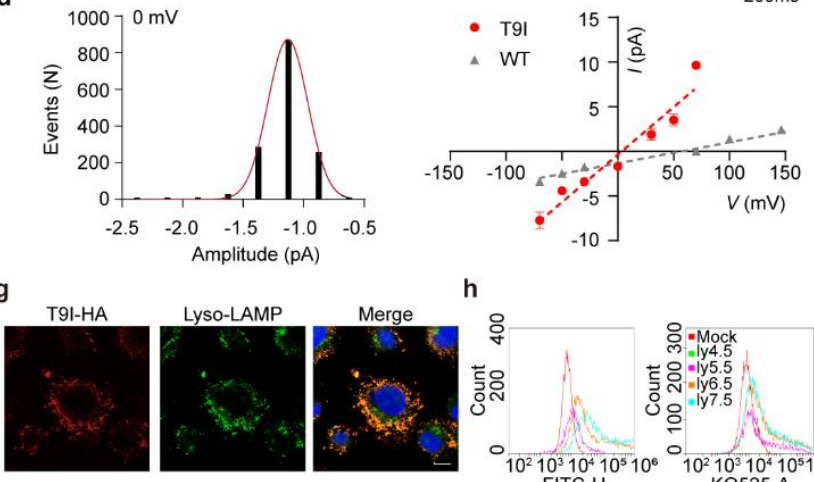

h

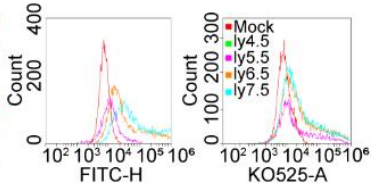

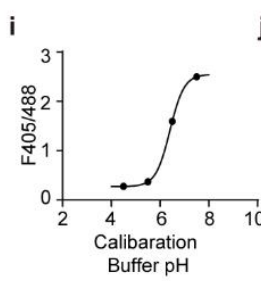
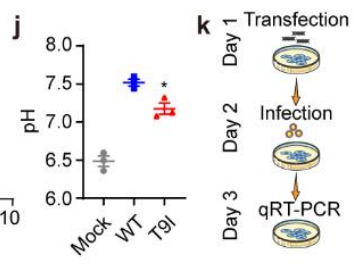

WT
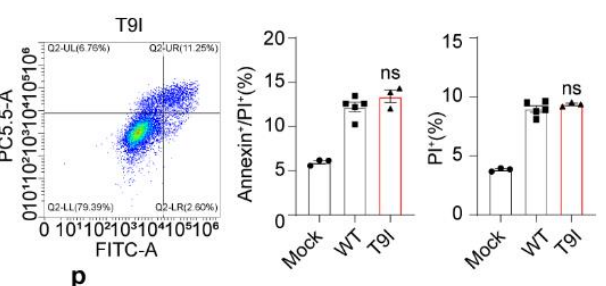

p
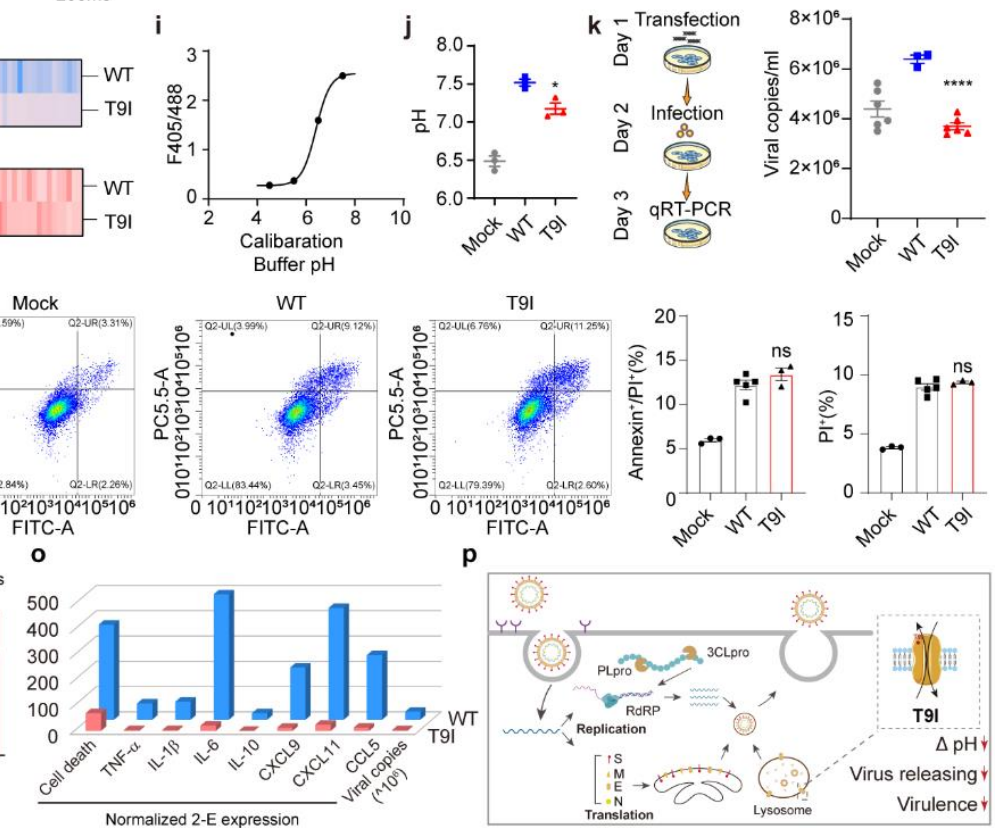

206 Fig.1 The envelope protein mutation T9I of SARS-CoV-2 Omicron variant shows

207 less pathogenicity and weakens virus production.

208 a, Structure of the 2-E TM domain ${ }^{10}$. Illustration of the amino-acid sequence of SARS-

209 CoV-2 E (8-38) and the indicated mutants. Threonine residues (T) are marked as blue and the $\mathrm{T}>\mathrm{I}$ mutants are marked as red. b, 2-E protein amino-acid sequence logo of 
SARS-CoV-2 Omicron variant. The I9 residues are marked as red. 2-E protein sequences were downloaded from China National Center for Bioinformation (CNCB) using the "Lineage Browse" option. Logos were generated using the WebLogo 3.7.4 online tool. c, Single-channel current recording of T9I after reconstitution in lipid bilayers with PC/PS $=3: 2$ lipids at indicated potentials and solutions (Left). d, Left, allpoint current histograms for the trace in $\mathbf{c}(0 \mathrm{mV})$ ("C" means Closed; "O" means Open). Right, $I-V$ curves of T9I in the solutions of $\mathbf{c}(\mathrm{n} \geq 3)$. e, Single-channel current recording in $500: 500 \mathrm{mM} \mathrm{KCl}$ solutions via proton titration result in different $\mathrm{pH}$ (cis or trans). Red arrow represents titration of $\mathrm{HCl}$. f, The heat map shows the transition of the currents from panel e under proton titration. g, Cell immunofluorescence of T9I (Red) and lysosome marker LAMP (Green). T9I is co-localized with lysosome. h-j, Lyso-pH indicator was used to assess the $\mathrm{pH}$ of the lysosome via flow cytometry. Flow cytometry pattern of the cells in buffers of known $\mathrm{pH}$ and contained ionophores to equilibrate the extracellular and lysosome luminal $\mathrm{pH}(\mathbf{h})$. Calibration curves were generated from data like those illustrated in $\mathrm{h}(\mathbf{i})$. j, The $\mathrm{pH}$ value of Vero E6 cells lysosome transfected with WT, T9I or Mock. k. Flow chart of the experiment (Left), the virus copies in supernatant determined by qRT-PCR assay (Right). MOI of SARS-CoV-2 for infection was 0.01. I, Protein expression levels of WT and T9I in Vero E6 cells after transfection (Up). The histogram shows the quantization of the result (Bottom). m, Flow cytometry pattern of Vero E6 cells transfected with WT or T9I plasmids. Right, flow cytometry analysis of Propidium iodide (PI) and Annexin V stained Vero E6 cells. n, Serum cytokine levels 
233 activity of WT, T9I in $\mathrm{pH}$ regulation, cell virulence, channel conductance and viral

234 copies normalized to the expression levels. p, Life cycle pattern diagram of SARS-

235 CoV-2 Omicron variant. T9I on envelope protein (2-E) of SARS-CoV-2 variant

236 Omicron forms a non-selective ion channel with weakened pathogenicity and virus

237 production. Experiments were independently performed thrice and similar results were

238 obtained. One set of reprehensive data is shown here $(\mathrm{n} \geqslant 3) .{ }^{*} p<0.05 ; * * p<0.01$;

$239 * * * p<0.001$; unpaired Student's t test. All error bars are SEM. 


\section{Method:}

\section{Plasmids and mutagenesis}

Wild type SARS-CoV-2-E sequences were synthesized by the Beijing Genomics Institute (BGI, China). The mutants 2-E ${ }^{\mathrm{T} 9 \mathrm{I}}$ sequence was generated by site-directed mutagenesis and confirmed by sequencing (BGI, China). Vector pET28a was used for protein purification; vector pcDNA5 and pcDNA3.1 were used for cell survival assay

247 and cell imaging.

\section{Cell culture and treatment}

Vero E6 and Raw 264.7 cells were grown in 90\% DMEM basal medium (Gibco, USA) supplemented with $10 \%$ fetal bovine serum (Gibco, USA) at $37^{\circ} \mathrm{C}$ under $5 \% \mathrm{CO}_{2}$.

\section{Planar lipid bilayers recording}

According to our previous methods, the purified protein $2-\mathrm{E}^{\mathrm{WT}}$ or $2-\mathrm{E}^{\mathrm{T} 9 \mathrm{I}}$ were incorporated into lipid bilayers to test their functionality. The lipid bilayers were prepared by PC: PS= 3: 2 (Avanti Polar Lipids, USA). Proteins were added in cis side and the solution was $50: 500 \mathrm{mM}$ (trans: cis) $\mathrm{KCl}$ or others, all solutions were buffered by $5 \mathrm{mM}$ HEPES, pH 6.35. Membrane currents were recorded by headstage connected with amplifier BC-535 (Warner Instruments, USA), filtered at 1-2 kHz and digitized by Axon Digidata 1440A (Molecular Devices, USA). The recording frequency was 10 kHz. The data were processed using pClamp 10.2 software (Molecular Devices, US). The single-channel conductance was determined by fitting to Gaussian functions (bin width $=0.25 \mathrm{pA})$. Opening time less than $0.5-1.5 \mathrm{~ms}$ was ignored to avoid noise 
flux equation:

$$
E_{m}=\frac{R T}{F} \ln \left(\frac{P_{\mathrm{Na}^{+}}\left[\mathrm{Na}^{+}\right]_{\text {out }}+P_{\mathrm{Cl}^{-}}\left[\mathrm{Cl}^{-}\right]_{\text {in }}+P_{\mathrm{K}^{+}}\left[\mathrm{K}^{+}\right]_{\text {out }}}{P_{\mathrm{Na}^{+}}\left[\mathrm{Na}^{+}\right]_{\text {in }}+P_{\mathrm{Cl}^{-}}\left[\mathrm{Cl}^{-}\right]_{\text {out }}+P_{K^{+}}\left[\mathrm{K}^{+}\right]_{\text {in }}}\right)
$$

\section{Cell viability and cytotoxicity assay}

Cell viability was measured by the CCK-8 kit (40203ES60, Yeason, China) according

Scientific Microplate Reader (Thermo Fisher Scientific Inc., USA).

\section{Western blotting and antibodies}

Proteins were resolved in $12 \%$ SDS-PAGE, transferred to PVDF membranes (GE,

USA). The PVDF membranes were incubated with primary antibodies against HA-Tag

(C29F4) (3724, CST, USA) or GAPDH (30201ES20, Yeasen, China), then incubated

with second antibodies against IgG (Yeasen, China).

\section{Flow cytometry of cell death}

After treatment, cells were detached, collected by centrifugation and resuspended in $1 \times$

V13242, Life, USA) and incubated at room temperature for $15 \mathrm{~min}$ in the dark.

279 Subsequently, samples were added $400 \mu \mathrm{L}$ of $1 \times$ binding buffer and kept on ice. Cells 


\section{Immunofluorescence microscopy.}

283

284

285

286

287

288

289

290

291

292

293

294

295

296

297

298

Vero E6 cells were seeded as $1 \times 10^{4}$ cells per well in coverslips on chamber slides (Thermo Fisher Scientific Inc., USA) overnight. Cells were transfected with WT or T9I with lysosome marker (LAMP1,). After transfection for $24 \mathrm{~h}$, cells were washed with phosphate-buffered saline (PBS) and fixed in 4\% paraformaldehyde for $10 \mathrm{~min}$ at room temperature. Then the cells were permeabilized in $0.5 \%$ Triton $\mathrm{X}-100$ in PBS for 3 min. The coverslips were washed twice with PBS and incubated with primary antibody in $1 \%$ bovine serum albumin (BSA) (36101ES25, Yeasen, China) for $2 \mathrm{~h}$ at room temperature. Anti-HA (HT301-01, Trans, China) and rabbit anti-LAMP (55273-1-AP, Proteintech, USA) were used to incubate the cells at a 1:1,000 dilution. Then, second antibodies FITC-AffiniPure Goat Anti-Rabbit IgG (33107ES60, Yeasen, China) and Cy3-AffiniPure Goat Anti-Mouse IgG (33208ES60, Yeasen, China)were used to incubate the cells at a 1:1,000 dilution. Images were captured using the Leica TCS-SP8 STED system with $100 \times$ oil objective.

\section{Virus loading and quantitative real-time PCR (qRT-PCR) analysis}

SARS-CoV-2 (nCoV-2019BetaCoV/Wuhan/WIV04/2019) was preserved at Wuhan institute of virology, Chinese Academy of Sciences. It was propagated and titrated with Vero E6 cells, and its associated operations were performed in a biosafety level 3 (BSL3) facility. Cell supernatants RNA was isolated with MiniBEST Viral RNA/DNA Extraction Kit (Takara, Japan) as described in the instruction, and cDNA was transcribed with PrimeScript ${ }^{\mathrm{TM}}$ RT reagent Kit with gDNA Eraser (Takara, Japan). In 

curve method on ABI 7500 (Takara TB Green Premix Ex Taq II, AK81975A) with a pair of primers targeting $S$ gene. The forward primer (5'-3') is:

CAATGGTTTAACAGGCACAGG; the reverse primer (5'-3') is:

\section{Determination of lysosome pH}

Vero cells were transfected with Lyso-pHluorin (addgene,70113) and 2-E plasmid.

After transfection, cells were trypsinzed and washed with Live Cell Imaging Solution

(LCIS). Then, replaced LCIS with cellular $\mathrm{pH}$ calibration Buffers containing $10 \mu \mathrm{M}$

USA) and incubate at $37^{\circ} \mathrm{C}$ for at least 5 minutes. At last, cells were analyzed via Flow cytometry with excited at $405 \mathrm{~nm}$ and $488 \mathrm{~nm}$, and the emission signals were collected with detection filters at 500 to $550 \mathrm{~nm}$ and 515 to $545 \mathrm{~nm}$, respectively. Flow cytometric data were collected and quantified using CytoExpert 2.4 software.

\section{Homology modeling}


324 homology models of the mutant envelope protein. This solid-state data yield to ten

325 lowest-energy structures. These ten structures were employed to construct ten

326 homology models of the mutant envelope protein using Modeller [A. Sali, T.L. Blundell,

327 Comparative protein modelling by satisfaction of spatial restraints, J Mol Biol 234

328 (1993) 779-815. 10.1006/jmbi.1993.1626.]. The models with the lowest root mean

329 square deviations from their template structures were selected.

\section{Statistics}

331 All measurements were derived from distinct samples. Statistics were performed in

332 GraphPad Prism. Statistical significance was determined by one-way ANOVA and two-

333 way ANOVA followed by pairwise Student's t-test with Tukey’s or Sidak's correction.

334 Two-tailed unpaired Student's t test was performed if only two conditions were compared. Data in the text is presented as Mean $\pm \mathrm{SEM}$. Adjusted P values are reported

336 in the figures. 


\section{Supplementary figure:}

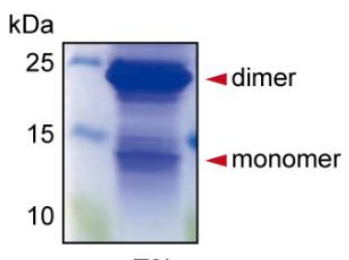

T9I

340 Supplementary information, Fig. S1 Purification of T9I proteins. Purification of

341 full-length 2-E ${ }^{\mathrm{T} 9 \mathrm{I}}$ protein with Ni-NTA affinity chromatography. 15\% SDS-PAGE gel

342 with coomassie blue staining.
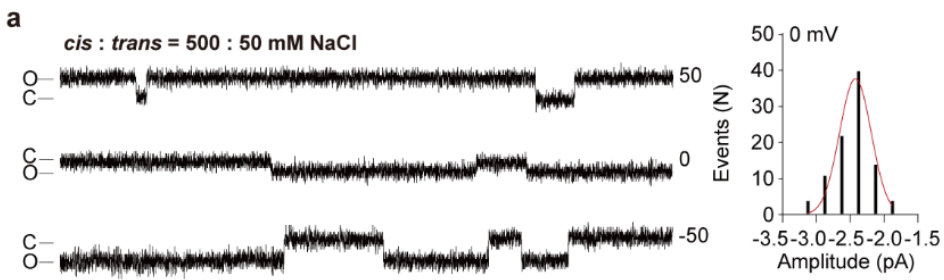

b cis : trans $=500: 50 \mathrm{mM} \mathrm{NaC}$
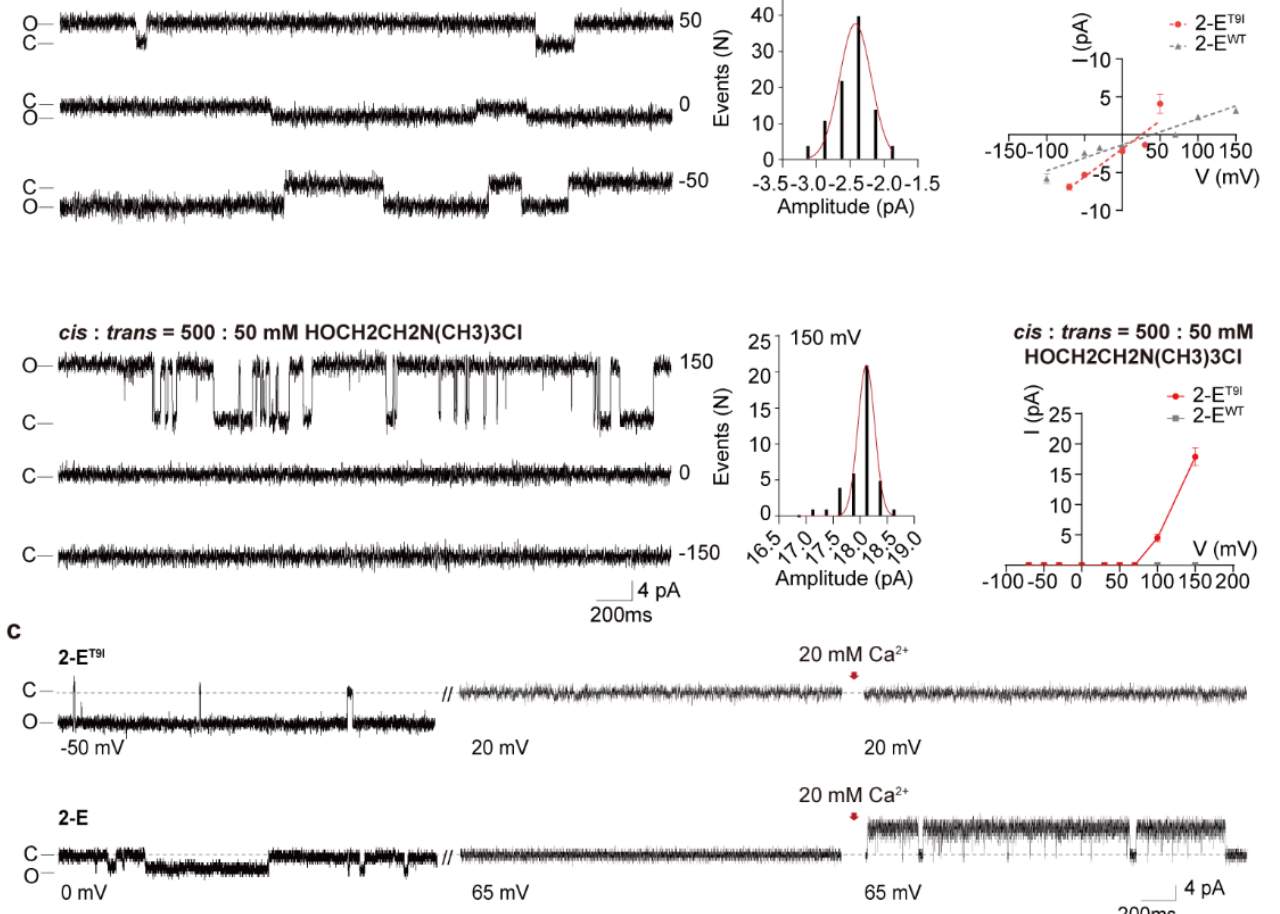

$20 \mathrm{mM} \mathrm{Ca}^{2+}$

$20 \mathrm{mV}$

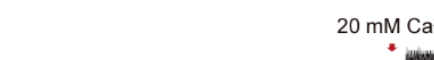

d
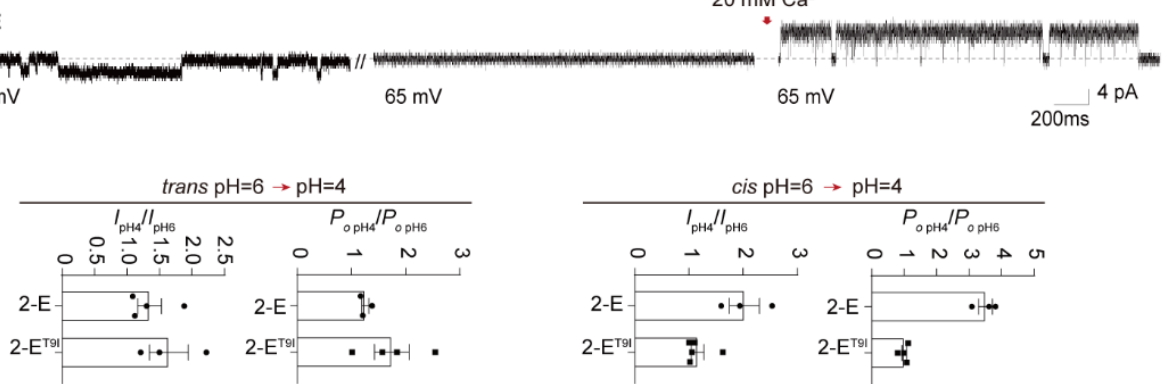

344 Supplementary information, Fig. S2 Electrophysiological property of T9I.

a. Single-channel current recording of T9I after reconstitution in lipid bilayers with 

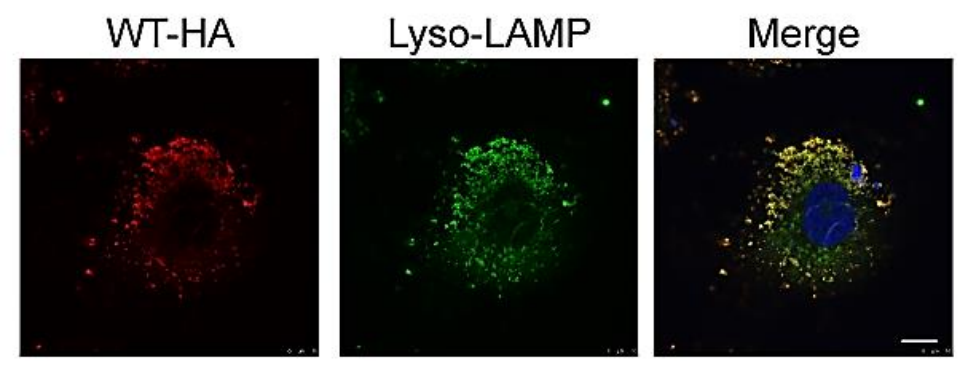

Supplementary information, Fig. S3 Wild type envelope protein (E) is co-localized

with lysosome. Cell immunofluorescence of WT (Red) and lysosome marker (Green).

Scale bar, $10 \mu \mathrm{M}$.
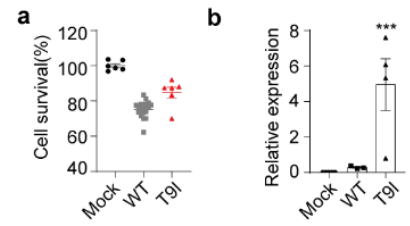

Supplementary information, Fig. S4 Cell viability of Vero E6 cells after andT9I. 
a

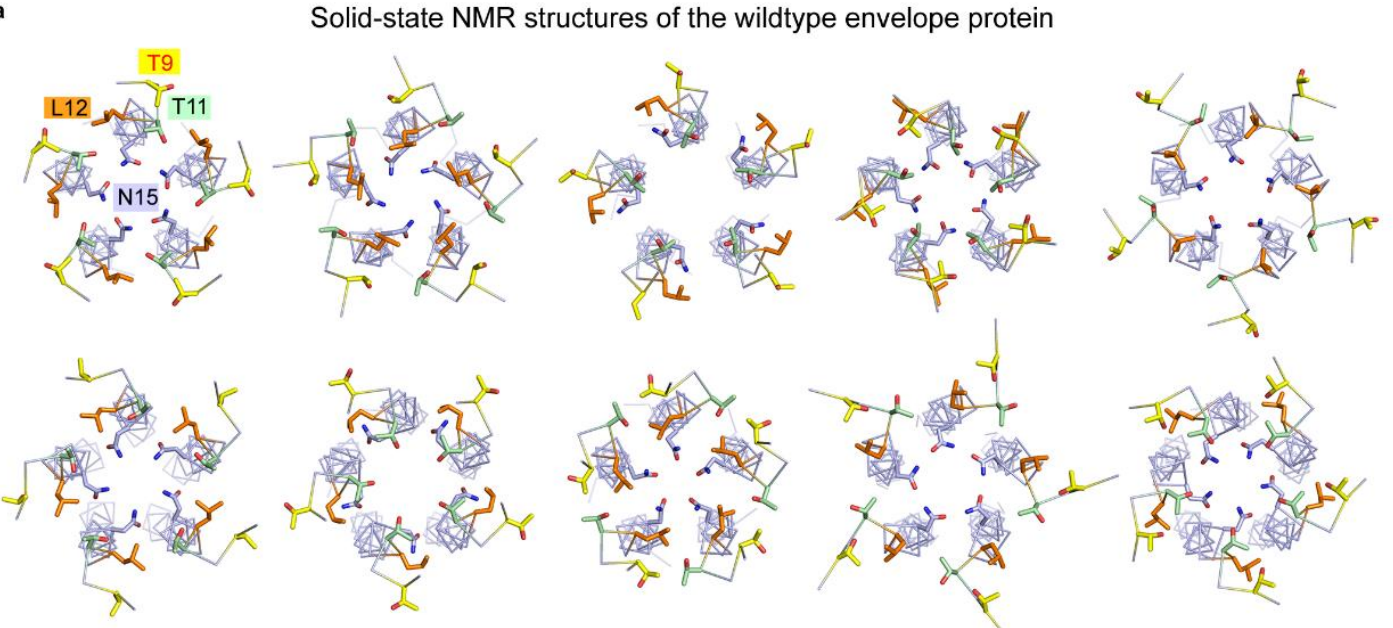

b

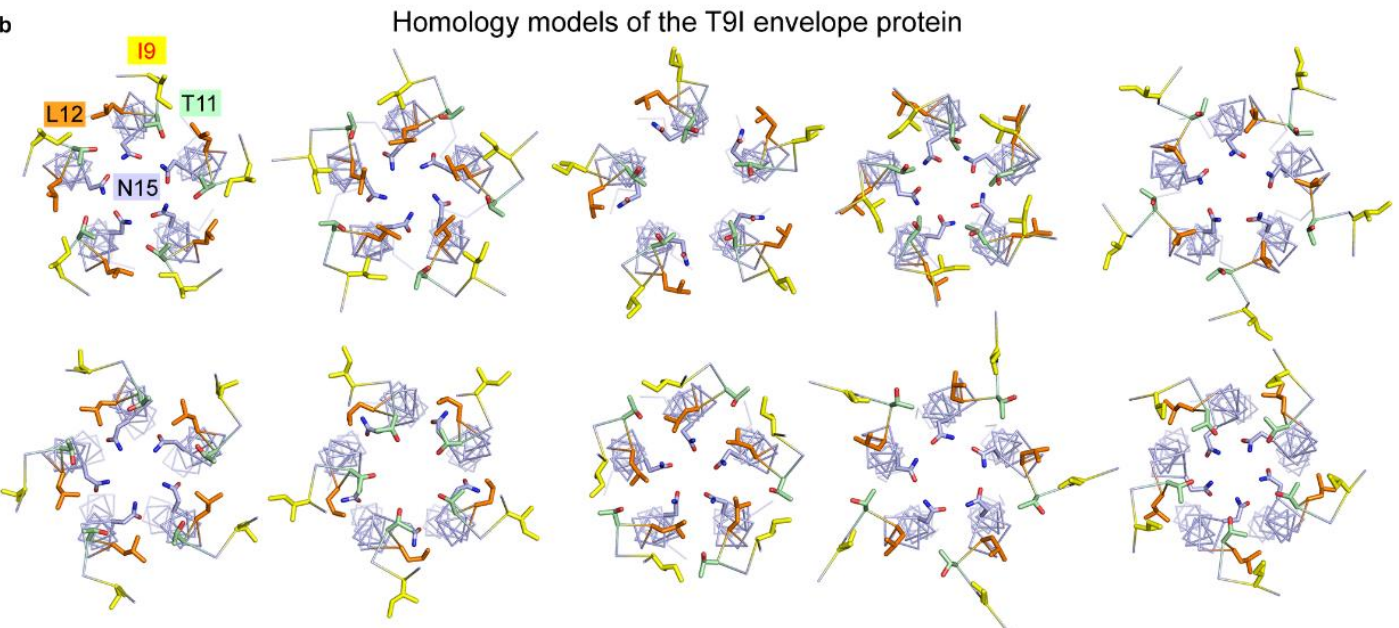
residue N15 are shown as sticks (T9 in yellow, T11 in green, L12 in orange, N15 in light blue). The protein is shown as ribbon. b, Top view of ten homology models of2$\mathrm{E}^{\mathrm{T} 9 \mathrm{I}}$, which built based on the solid-state NMR structures of wildtype SARS-CoV-2 
372 envelope protein (PDB code: 7k3g). Three N-terminal residues I9, T11, L12 and a pore-

373 lining residue N15 are shown as sticks (I9 in yellow, T11 in green, L12 in orange, N15

374 in light blue). 